\title{
La Faculté de médecine de l'Université de Lausanne (1890): un pari politique
}

par Guy Saudan

\section{RÉsumé}

La création de la Faculté de médecine de l'Université de Lausanne relève davantage de sollicitations politiques que médicales: la souveraineté cantonale autorise le projet d'une école, en 1804, et le fédéralisme vaudois provoque sa réalisation, en 1890.

Dans le dernier quart du XIX $X^{e}$ siècle, Lausanne doit se tailler sa place entre Berne, la puissance patricienne promue capitale fédérale, et l'industrieuse Genève. Le débat cristallise autour du tracé des chemins de fer et de la localisation des études supérieures: le chef-lieu vaudois sera carrefour ferroviaire et ville universitaire. Les libéraux sécularisent l'Académie et les radicaux y font pénétrer les sciences. L'ouverture d'une Faculté de médecine à Genève (1876) et l'institution d'examens fédéraux (1877) font office de catalyseurs : précédée d'un enseignement propédeutique (1881), une Faculté complète de médecine voit le jour à Lausanne, permettant la transformation de l'ancienne Académie en Université (1890).

Le $\mathrm{XIX}^{\mathrm{e}}$ siècle, engendrant le Canton de Vaud, produit aussi et surtout la médecine moderne: synchronisme fécond, réitérant celui de la Renaissance médicale et du Régime bernois en Pays de Vaud (1536-1798). Dès 1804, un an à peine après la naissance de l'Etat de Vaud, François Verdeil (17471832), chef du Bureau de santé, demande au Gouvernement l'institution d'une école de médecine, tirant exemple de Zurich. Initiative perspicace autant que prématurée car le Canton ploie sous le fardeau de l'indépendance, et l'Académie héritée de Berne n'est qu'un séminaire de pasteurs.

\section{Initiatives prématurées}

Est médecin qui veut, en Pays de Vaud, jusqu'à la fin du XVIII ${ }^{\mathrm{e}}$ siècle, faute de réglementation professionnelle. Aussi la formation universitaire des médecins vaudois est-elle peu fréquente, sans parler de son coût. Les rares 
candidats se rendent à Bâle, Montpellier, Paris, Strasbourg ou Leyde, éventuellement en Italie ou Grande-Bretagne. Pour qui en a les moyens, il convient alors de suivre les cours d'au moins trois établissements étrangers, selon l'usage de la "pérégrination académique». En Suisse, Bâle seule dispose d'une Faculté de médecine, fondée avec l'Université (1460). Puis, à Zurich, un petit groupe de médecins et chirurgiens ouvrent un Medizinischchirurgisches Institut (1782), bientôt repris par le Canton (1804) et transformé en Faculté à la création de l'Université (1833). Même scénario à Berne (1798), puis transformation en Faculté dans le cadre de l'Académie (1805) et enfin de l'Université (1834).

A Lausanne, outre l'initiative de Verdeil, médecin d'origine languedocienne et patriote vaudois de la première heure, le bailli Gabriel-Albert d'Erlach, président du Collège de médecine de Lausanne, organe de surveillance des affaires médicales instauré en 1787, conduit en fait par Auguste Tissot (1728-1797), écrit à Leurs Excellences, en 1788 déjà :

«Je prends la liberté de remarquer qu'il est impossible d'aider à la profession médicale tant qu'il n'y aura pas dans le pays une Ecole de chirurgie et d'anatomie»'.

Un enseignement médical officiel, quoique temporaire, se fait jour à Lausanne dès le début du XVIII ${ }^{e}$ siècle, lorsque Pierre-François Martin (v. 16981756) procède, à l'Hôtel-de-Ville, à des «anatomies publiques», sous la haute autorisation et inspection de l'Académie, avec l'assentiment des autorités locales et l'appui de Leurs Excellences (1731-1740) ${ }^{2}$. Une seconde étape, psychologique, est franchie avec la nomination de Tissot à l'Académie, comme premier «professeur public en médecine», titre purement honorifique obtenu de Berne par le grand Haller, sans consultation de l'Académie, afin de retenir l'intéressé au pays $(1766)^{3}$. En fait, le premier enseignement officiel régulier est donné par Jean-André Venel (1740-1791), d'origine languedocienne comme Verdeil, non docteur, créant à Yverdon la première école de sages-femmes de Suisse (1778) ${ }^{4}$.

Suite à la requête de Verdeil, le Grand Conseil vaudois crée deux chaires à l'Académie, l'une de médecine, l'autre de chirurgie (1806). Au printemps 1812 enfin, la chaire d'anatomie et de chirurgie est mise au concours. Mais personne n'est nommé et le projet sombre dans l'oubli ${ }^{5}$. Le jeune Etat de Vaud doit, à ce moment, faire face en priorité à l'œuvre législative considérable qu'impliquent la construction de routes, l'organisation d'une police de santé, la conduite des secours publics, sans parler de la régie des postes, des autres questions agricoles, religieuses ou politiques. Dans ce contexte, 
aucune nécessité à proprement parler nouvelle et impérieuse n'appuyant le projet de Verdeil, il est condamné d'avance. En outre, l'Académie, venant tout juste d'accorder l'autonomie à la physique et aux mathématiques à la fin du XVIII ${ }^{\mathrm{e}}$ siècle, n'est pas prête à recevoir les sciences à bras ouverts. Surtout, la médecine elle-même n'est pas mûre à Lausanne: organisation sanitaire nouveau-née, services hospitaliers proprement dits inexistants, profession médicale à constituer.

\section{Organisation sanitaire}

En Pays de Vaud, médecins titrés et autodidactes, scientifiques et empiriques, s'affrontent tout au long du $\mathrm{XVIII}^{\mathrm{e}}$ siècle. C'est que l'exercice de la profession y est totalement libre: situation anarchique contrastant avec la réglementation genevoise, pour ne rien dire de l'examen des praticiens introduit en Occident dès 1140, dans le Royaume de Naples et Sicile. Seuls n'éprouvent pas la nécessité d'une réforme les empiriques eux-mêmes et le peuple. Parmi les médecins gradués, Jacob Constant, le premier, soulève la question en 1692, dans ses Observationes medicae. Pierre-François Martin, chargé de représenter ses collègues auprès de Berne, reprend l'argumentation dès 1730, comme en témoignent ses lettres à Haller. Dans le Mercure suisse, le plus important périodique helvétique de langue française, il oppose, en 1735, le «vrai médecin» à l' «empirique» ou «charlatan». Dans son Avis au peuple, tout entier dirigé contre la superstition, Auguste Tissot consacre un chapitre bien connu aux «charlatans et maiges» (du patois «maidzo», celui qui soigne), dressant un véritable réquisitoire, sans pour autant demander explicitement l'intervention du gouvernement. Art médical et pouvoir politique unissent néanmoins leurs efforts en 1765, par la publication d'un mandat de Leurs Excellences, visant à conférer à la pratique médicale en général une base légale, valable dans tout le pays ${ }^{6}$. Cet acte fondamental traduit la conjonction nouvelle d'une certaine confiance de la médecine en son savoir et de l'Etat en son pouvoir, inaugurant une politique de santé publique quittant le cadre strict de la lutte contre les épidémies. Le Conseil de santé de Berne, en effet, avait été définitivement établi en 1709 contre la contagion, à l'instar de ses homologues alémaniques et prédécesseurs italiens. C'est de qualité des soins et de réglementation professionnelle qu'il doit désormais se préoccuper avant tout. Mais le décret de 1765 reste impuissant à renverser le courant séculaire de la confiance d'un 
peuple en ses maiges. Aussi réclame-t-on de plus belle l'instauration d'une patente pour l'exercice de la médecine. Après un ultime mandat de portée générale consacré à ce problème en 1785, Leurs Excellences se décident à créer, en 1787, le Collège de médecine de Lausanne. A cette institution incombent l'inspection générale de tous ceux qui pratiquent l'art de guérir, le contrôle des maladies contagieuses, la surveillance des vendeurs de drogues, la détection des abus survenant dans l'exercice de la médecine, mais en fait surtout l'examen des praticiens dépourvus de titres à la date de son entrée en fonction. Au début de 1788 sont édictés les règlements fixant le code professionnel des médecins, chirurgiens et apothicaires; pour les sagesfemmes, on se satisfait de l'existence de l'école fondée par Venel. Avant cette date, comme le souligne Eugène Olivier, «rien n'avait été entrepris pour doter le pays de médecins capables. Ceux-ci ne se trouvaient que dans les villes. Partout ailleurs la grande masse du peuple n'eût su à qui recourir sinon au maige ${ }^{7}$.

La volonté de distinguer les scientifiques des empiriques est également fondamentale dans le projet d'école de médecine de Verdeil : «Ce serait le vrai moyen de délivrer le pays de cette foule de maiges qui l'infestent» ${ }^{8}$.

Mais le Collège de médecine s'attelle déjà à d'autres tâches que la réglementation professionnelle, non moins considérables, à commencer par la transformation de l'hôpital en établissement pour malades.

\section{«Naissance de la clinique»}

L'Hôpital Notre-Dame de Lausanne, fondé en 1282, rebâti au XIV ${ }^{\mathrm{e}}$ siècle, entièrement reconstruit en 1767-1771, n'évolue guère dans son principe, de 1300 à 1800 : comme son nom l'indique - Hospitale pauperum -, il s'agit d'un asile, recueillant pêle-mêle indigents, délinquants, passants (pèlerins), infirmes et, le cas échéant, malades. C'est que l'Hôpital de l'Isle, à Berne, est accessible aux Vaudois, avec son service médical régulier, souvent mentionné au $\mathrm{XVI}^{\mathrm{e}}$ siècle. Mais, à Lausanne, les seuls établissements exclusivement destinés aux patients sont les maladières pour pesteux, lépreux et autres contagieux, désaffectées dès le début du XVII ${ }^{\mathrm{e}}$ siècle: jusqu'en 1800, aucun hôpital ne dispose de ce que nous appellerions aujourd'hui un service hospitalier. La reconstruction intégrale de l'Hôpital en 1771 correspond en fait à une simple rénovation architecturale. La piètre utilisation du nouveau bâtiment est dénoncée en vain à plusieurs reprises ${ }^{9}$. Cependant, Hospice 


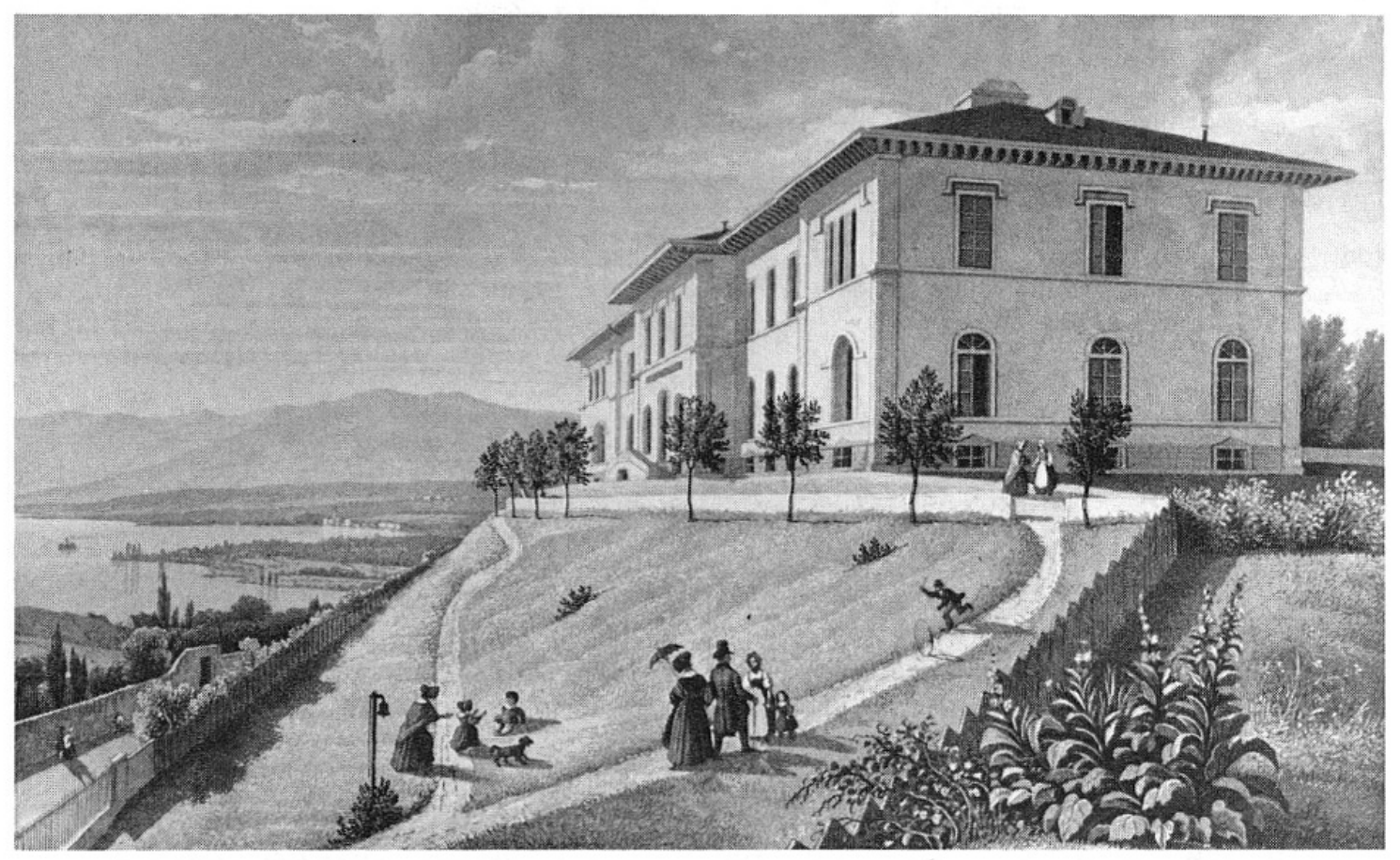

L'Asile des aveugles de Lausanne (1843), vers 1845 (Gravure de Frédéric Martens, Musée historique de Lausanne).

cantonal dès 1806 , assuré d'une dotation considérable grâce à la suppression de l'Hôpital de Villeneuve, l'établissement entame sa lente mue de «miséricorde» en hôpital moderne, avec le départ successif des aliénés $(1811)^{10}$, des détenus (1826) et des établissements de redressement pour garçons (1847) et filles $(1869)^{11}$. La transformation de l'asile en centre de soins s'étend donc sur quatre-vingts ans, du rapport Tissot à l'ultime épuration. Mue trouvant son expression décisive dans le dernier quart du siècle, avec deux premières réalisations publiques d'envergure: l'Asile d'aliénés de Cery $(1873)^{12}$ et l'Hôpital cantonal du Calvaire (1883). L'Etat de Vaud, jusque-là trop absorbé par les charges multiples de l'indépendance et les soubresauts politiques de 1830, 1845, 1861, est enfin en mesure d'assumer son rôle en matière d'établissements pour malades. De plus, l'hôpital général, après six siècles au cœur de la cité, domine désormais symboliquement la ville, du haut de la pente du Champ-de-l'Air.

Mais, sans attendre ces aboutissements du secteur public, c'est l'initiative privée qui assure l'avènement de la clinique à Lausanne, durant les années 1840-1880, dans l'esprit du Réveil, mouvement religieux d'origine anglosaxonne, d'essence romantique, prônant un christianisme actif. Relevons la 


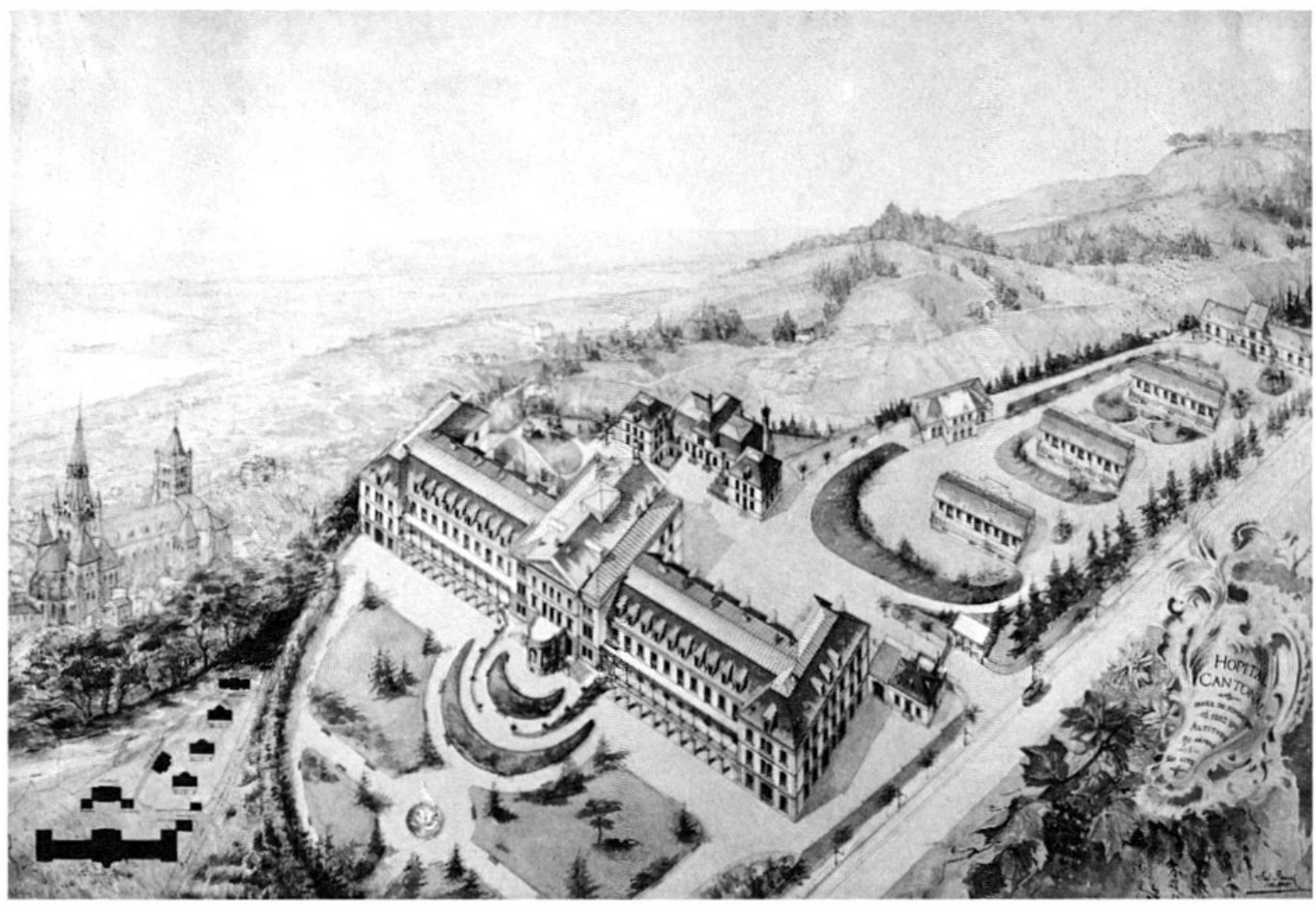

L'Hôpital cantonal de Lausanne et ses dépendances (1883), en 1896 (Dessin aquarellé de Jacques Regamey, CHUV/Lausanne).

fondation de l'Asile des aveugles (1843) ${ }^{13}$ et de l'Hospice de l'enfance $(1861)^{14}$, premiers du genre en Suisse, ainsi que de l'Hospice orthopédique de la Suisse romande, héritier de la tradition venélienne $(1876)^{15}$. Dans le domaine ambulatoire, il faut signaler le Dispensaire central (1887), bientôt transformé en Policlinique universitaire $(1892)^{16}$. Enfin, concernant les soins, l'Institution des diaconesses de St-Loup, initialement établie à Echallens, est la première de Suisse $(1842)^{17}$; l'Ecole normale évangélique de gardes-malades indépendantes (1859), ultérieurement dénommée La Source, constitue quant à elle une première mondiale ${ }^{18}$.

Les couches aisées de la population, en particulier les étrangers en séjour, toujours plus nombreux, se font soigner à domicile ou à l'hôtel, s'épargnant la honte, voire la terreur de l'hospitalisation. A partir de 1860, les établissements de luxe se multiplient dans la cité lémanique, grâce au chemin de fer notamment; avant 1914, le Beau-Rivage Palace à Ouchy est transformé en véritable clinique, symbole d'un «tourisme médical» retentissant jusqu'à nos jours sur l'heureux développement de la médecine à Lausanne. 


\section{Profession médecin}

La profession médicale s'affirme au $\mathrm{XIX}^{\mathrm{e}}$ siècle, entre la réglementation tardive de 1788 et l'instauration d'examens fédéraux en 1878. Une Société vaudoise des sciences médicales - future Société vaudoise de médecine - naît en 1829, dix ans après celle des sciences naturelles, sous la houlette de Mathias Mayor (1775-1847), puis Jean de La Harpe (1802-1877) ${ }^{19}$. En 1867, une Société médicale de la Suisse romande est fondée à Lausanne, dans le but de promouvoir l'esprit de corps, la recherche scientifique et les stages à l'étranger. Hormis les éphémères et néanmoins remarquables Mémoires de la Société des sciences physiques de Lausanne (1783-1788), où paraît en son temps le seul écrit orthopédique de Venel, la Société vaudoise de médecine ne dispose d'un organe périodique qu'en 1866, vite relayé par le Bulletin de la Société médicale de la suisse romande (1868-1880), devenant Revue médicale de la Suisse romande. Auparavant, dès 1842, les travaux médicaux sont publiés dans le Bulletin de la Société vaudoise des sciences naturelles.

Dans la seconde moitié du siècle prennent naissance, à Lausanne et dans le canton, de véritables dynasties médicales: les Cérésole, Dufour, Guisan, Larguier, Muret, Rossier et tant d'autres ${ }^{20}$. Vers 1900 , la conjonction de la médecine scientifique, de l'ère industrielle et de la société urbaine achève d'identifier la profession médicale au modèle même de la profession moderne: élitisme, études exigeantes, diplôme palpable, niveau de vie élevé, prestige social, corporatisme influent, image publique plus que favorable. Toutefois, si la catégorie des omnipraticiens est largement représentée à Lausanne, celles, nouvelles, des chercheurs de laboratoire et des consultants hospitaliers ou universitaires doivent le plus souvent exercer ailleurs. A cet égard, trois grands noms viennent aussitôt à l'esprit: Alexandre Yersin (1863-1943), bactériologiste «assis» à Paris et «debout» en Indochine, découvreur du bacille de la peste ${ }^{21}$; Auguste Forel (1848-1931), privat-docent à Munich, professeur à Zurich, fondateur de la psychiatrie suisse ${ }^{22}$; Gustave Roussy (1874-1948), fondateur de l'Institut du cancer de Villejuif, à Paris ${ }^{23}$. A l'évidence, le Pays de Vaud manque alors d'institutions d'enseignement et de recherche.

\section{Fédéralisme}

Verdeil exprime en 1804 le besoin médical d'une école de médecine; vers la fin du siècle, le Conseiller d'Etat Eugène Ruffy (1854-1919) et le Conseiller 
communal Marc Dufour (1843-1910) ${ }^{24}$, ophtalmologue, en manifestent l'exigence politique.

La Suisse de 1880, Etat fédéral fondé par la Constitution de 1848 révisée en 1874, est sur la voie de l'intégration nationale. D'où divers mouvements, centrifuges autant que centripètes. En réaction au projet - jamais aboutid'Université fédérale, stimulées par le renouveau libéral des hautes écoles, les académies protestantes de Zurich (1833), Berne (1834), puis Genève (1873) se transforment en Universités, portant à quatre avec Bâle le nombre de ces institutions dans le pays, entraînant la création d'autant de Facultés de médecine. Tendance centripète, en revanche d'une majorité des médecins quant à l'exercice de leur profession, lesquels éprouvent l'urgente nécessité d'abattre les frontières cantonales. En ce sens, la plupart des cantons, romands exceptés, se rallient à un Concordat (1867) ${ }^{25}$. La Loi fédérale du 19 décembre 1877 tranche la difficulté: des examens fédéraux sont institués.

Faculté de médécine à Genève (ouverte en 1876), examens unifiés imposés par la Berne fédérale: Vaud, après des années de résistance passive de ses autorités sanitaires, doit agir. Comme le rappelle Gustave Delay, professeur de policlinique médicale à Lausanne et chef du Service sanitaire cantonal:

«La Faculté de médecine de l'Université de Lausanne est d'origine fédéraliste. Il faut être Suisse pour comprendre la portée de ce terme» ${ }^{26}$.

Une Section propédeutique des sciences médicales est ouverte en 1881 à la Faculté des sciences de l'Académie. Ainsi Lausanne devient-elle le siège d'examens fédéraux et la Faculté des sciences peut-elle compter sur le maintien de sa fréquentation. Il était à craindre, en effet, que les candidats médecins ne la désertent, si elle devait se cantonner aux sciences naturelles, celles-ci et les sciences anatomophysiologiques étant examinées simultanément, suivant la nouvelle réglementation. Le premier pas vers une Faculté de médecine à Lausanne est donc, en quelque sorte, obligé, compte tenu de l'étape précédente, soit l'entrée des sciences à l'Académie. A ce sujet, un profond remaniement de la Haute Ecole s'opère tout au long du siècle, au gré d'adaptations politiques répétées : à cinq constitutions cantonales successives $(1803,1831,1845,1861,1885)$ correspondent cinq lois régissant l'Académie $(1806,1837,1846,1869,1890)$. Celle de 1837, œuvre des libéraux, sécularise l'institution en établissant trois Facultés: Théologie, Droit, ainsi que Lettres et sciences. Louis Ruchonnet (1834-1893), chef du Département de l'instruction publique, réorganise l'Académie dans une perspective universitaire, dédoublant la Faculté des lettres et sciences (1869). Le courant 
libéral et scientifique gagne du terrain et les dirigeants radicaux ne laissent échapper aucune bonne occasion de l'amplifier. La fermeture de l'Ecole de pharmacie de Strasbourg, suite à l'annexion de l'Alsace à l'Allemagne, favorise l'ouverture à Lausanne d'une institution analogue (1873); l'instauration d'examens fédéraux de médecine impose la mise sur pied d'un enseignement propédeutique (1881); enfin, la disponibilité d'une forte somme d'argent - le legs de Rumine - permet de «compléter» l'enseignement de la médecine à Lausanne (1890). L'exécution du testament de Gabriel de Rumine (1841-1871), ingénieur lausannois d'origine princière russe, prématurément décédé ${ }^{27}$, fournit en effet l'occasion inespérée en 1886 , de régler la question des bâtiments universitaires, pendante depuis 1869. La Ville de Lausanne, destinataire de ce legs de quelque trois millions de francs, signe le 23 août 1888 une convention avec l'Etat, au terme de laquelle la somme est mise à disposition du Canton, moyennant quoi celui-ci s'engage à «transformer» l'Académie en Université, par la création d'une Faculté de médecine complète, opération accomplie avec la Loi du 10 mai 1890, signée d'un grand homme d'Etat, digne successeur de Ruchonnet, Eugène Ruffy.

Dès fin 1887, ce dernier réunit une très active Commission préconsultative pour la création d'une Faculté de médecine ou «Commission Ruffy», chargée notamment d'établir un budget de fonctionnement, composée de six médecins: Edouard Bugnion, Edouard de Cérenville et Marc Dufour - tous trois libéraux-conservateurs, dans la quarantaine -, Emile Dind, Frédéric Paschoud et César Roux - radicaux, dans la trentaine ${ }^{28}$. La moyenne d'âge est de trente-six ans et demi. Pour sa part, Ruffy, chef du Département, a trente-trois ans. Trois des membres de la Commission enseignent déjà la médecine à l'Académie: Dufour l'hygiène en Sciences, Roux la médecine légale en Droit et Bugnion l'anatomie à la Section propédeutique. Le moment venu, les six sont appelés par Ruffy à enseigner à la nouvelle Faculté: seul le psychiatre Paschoud décline l'invitation.

L'ophtalmologue Marc Dufour, praticien de renommée internationale, premier doyen de la future école, joue un rôle de premier plan. Intime de Rumine, il est rapporteur, le 7 mars 1887, devant le Conseil communal de Lausanne, de la Commission chargée de l'affectation du généreux legs:

«Ce n'est pas toujours dans les écoles les plus grandes, ni au pied des hommes les plus célèbres qu'on travaille le mieux», expose-t-il. «Dans quelques branches des sciences médicales, il ne suffit pas d'entendre les choses dans un grand auditoire, ni de cultiver son esprit seulement, mais il faut éduquer les sens. Or, cette éducation requiert une pratique individuelle souvent renouvelée, qu'il est plus facile d'acquérir dans une petite que dans une 
grande école. Les écoles modestes ont donc certains avantages positifs, et ne présentent d'inconvénients que pour ceux des étudiants qui pensent borner à elles le cycle complet de leurs études.»

\section{S'agissant de la transformation de l'Académie, Dufour ajoute:}

«Il en ressortirait, nous le croyons, une prospérité augmentée de la ville et de la campagne et, chose plus précieuse encore, une certaine valeur intellectuelle et morale à notre cité. Cette valeur nous assignerait un rang supérieur à celui qui nous serait donné par notre activité passée et par le chiffre de notre population.»

\section{Et il conclut :}

«Rien n'est plus propre à élever le peuple d'une cité, ou celui d'un petit Etat, que de poursuivre la réalisation du progrès dans le domaine de l'intelligence. Etre inspiré par la recherche de la beauté dans l'art, de la vérité dans la science, être poussé par les idées plus que par les intérêts, que les idées soient religieuses, littéraires, scientifiques ou politiques, voilà ce qui fait qu'une génération marque son passage dans l'histoire» ${ }^{29}$.

L'attitude idéaliste et l'argument de la modestie de l'institution synonyme de qualité ne doivent pas masquer l'exigence primordiale en faveur du développement de la Haute Ecole et de Lausanne, le pari politique: soutenir la comparaison avec les villes universitaires de Suisse.

Eugène Ruffy souligne, pour sa part, l'avantage moral d'une formation autochtone :

«Il n'est pas utile que le jeune homme quitte trop tôt son pays pour aller étudier au dehors. Il est bon qu'il forme auparavant son caractère, qu'il acquière des principes et des opinions. Puis, plus tard, il ira chercher le complément d'instruction qui lui sera nécessaire» ${ }^{30}$.

Il est facile d'opposer cette prise de position au point de vue exprimé par Tissot un siècle auparavant :

«S'il fallait statuer sur le lieu des études, je statuerais que les habitants où il y a une école de médecine ne pourraient pas l'y étudier. Cette facilité de l'étudier sans frais, sans déplacement, sans peine; cette confiance d'y trouver plus de patronage dans les épreuves qu'ailleurs, déterminent une foule de jeunes gens, qui ne sont qualifiés en aucune façon pour cette vocation, à s'y vouer: les rues sont couvertes de docteurs titulaires, et les malades trouvent à peine un bon médecin. Il en est de même pour les autres études: il faut bien se garder de les rendre trop peu coûteuses, parce qu'alors les Académies, les Universités se remplissent de sujets qui devraient labourer les champs et fossoyer les vignes» ${ }^{31}$.

Tissot entend protéger la profession, Ruffy le pays.

La question de la localisation des études supérieures constitue, avec celle du tracé des chemins de fer dans la seconde moitié du XIX ${ }^{\mathrm{e}}$ siècle, l'un des deux débats d'intérêt crucial pour le développement de la ville. Dans chacun 
des cas, Lausanne emporte la décision en débloquant une forte somme, sans appui financier du Canton: ligne Genève-Berne par Lausanne-Oron-Fribourg (1856) ${ }^{32}$ et Convention pour la transformation de l'Académie (1888). 1890 enregistre à la fois la création de l'Université de Lausanne et la Fusion des compagnies ferroviaires Suisse occidentale-Simplon et Jura-BerneLucerne, en vue du percement du Simplon. D'ailleurs, le Palais de Rumine et le Tunnel du Simplon sont significativement inaugurés conjointement en 1906: Lausanne devient carrefour ferroviaire et ville universitaire.

Tant pour les chemins de fer que pour l'Université - projets de lignes Genève-Berne et Paris-Milan ignorant Lausanne; Faculté de médecine au bout du lac et examens fédéraux -, Berne et Genève sont perçues, à plus au moins juste titre, comme rivales, sinon adversaires de Lausanne, dans le contexte passionné de l'indépendance vaudoise récente et du long débat des années 1860/70 opposant, autour de la révision de la Constitution helvétique, centralisme et fédéralisme.

\section{Transformation de l'Académie}

La Convention sur l'affectation du legs de Rumine (1888), base de la création de l'Université, est adoptée au Grand Conseil à l'unanimité des votants, justement saluée par le chef du Département de l'instruction publique, à l'ouverture des cours, le 22 octobre 1890:

\footnotetext{
«Dans ce pays avant tout agricole, il s'est trouvé dans une assemblée législative, dont la plupart des éléments sont très éloignés de notre centre d'instruction supérieure, une unanimité magnifique pour voter la création d'une Université et pour assumer la responsabilité des sacrifices qu'une pareille création entraîne» ${ }^{33}$.
}

Adhésion de la rue également, dans une ville décorée et pavoisée : du 18 au 20 mai 1891 se déroulent les Fêtes inaugurales de l'université, dans la liesse populaire et la ferveur patriotique.

Pourtant, l'enthousiasme n'est pas absolument général. Tandis que les représentants de la Haute Ecole genevoise raillent les ambitions universitaires lausannoises, craignant en particulier pour la fréquentation de leur propre Faculté de médecine ${ }^{34}$, des milieux paysans vaudois lancent une pétition anti-universitaire, appuyée par divers journaux locaux: le Démocrate de Payerne, le Courrier de la Broie d'Avenches, le Progrès de Châteaud'Ex, le Messager des Alpes d'Aigle. Quelque 2000 signatures seulement sont récoltées et le mouvement fait long feu ${ }^{35}$. Plus redoutable, l'opposition 
diffuse et influente des milieux médicaux, brandissant le spectre de la pléthore professionnelle. Frédéric Recordon lui-même (1811-1889), père des hôpitaux modernes de Lausanne, chef du Service sanitaire cantonal, professeur honoraire à l'Académie et de surcroît vice-président du Comité directeur des examens fédéraux, s’exprime ainsi, en 1881 déjà :

«Cette prétention de créer une chaire d'anatomie et de physiologie est réellement ridicule, pour ne rien dire de plus» ${ }^{36}$.

Quant à Eugène Olivier (1868-1955), candidat médecin à Lausanne, futur et fameux historien de la médecine et de la santé en Pays de Vaud, il contresigne carrément la pétition opposée à la transformation de l'Académie:

«Ma volée inaugura l'enseignement clinique à la Faculté de médecine de Lausanne en 1890 et participa aux Fêtes universitaires du printemps suivant, célébrant l'étape nouvelle franchie par la vieille Académie de 1536 [sic]. Transformation qui ne paraissait à beaucoup ni désirable, ni justifiée. Des pétitions circulèrent, qui protestaient contre le projet; si ces feuilles ont été conservées, on y trouverait mon nom. Après quoi, une fois la décision prise, les opposants n'en furent pas moins reconnaissants de pouvoir continuer leurs études à Lausanne; et ceux qui firent la comparaison avec les universités allemandes purent voir que chez nous, sans installations grandioses, il se faisait un travail aussi sérieux, grâce au fait que les professeurs, ayant dix fois moins d'étudiants à diriger, pouvaient les suivre de près» ${ }^{37}$.

Mais sciences et médecine sont maintenant solidement implantées à l'Académie. A la Section propédeutique des sciences médicales, on appelle en 1881 les professeurs Edouard Bugnion (1845-1939: anatomie) et Alexandre Herzen (1839-1906: physiologie), puis en 1885 Nathan Loewenthal (1855-1942: histologie). Bugnion, d'une famille de banquiers lausannois, beau-frère d'Auguste Forel, entomologiste distingué, étudie à Paris, Munich et Zurich, avant d'enseigner l'anatomie, l'histologie et l'embryologie à l'Institut vétérinaire de Berne. Herzen et Loewenthal sont disciples du physiologiste allemand Moritz Schiff, établi à Genève. Herzen, russe d'origine, naturalisé à Fribourg, fils de son célèbre homonyme aristocrate révolutionnaire, professeur à l'Institut des études supérieures de Florence, est un «moniste» affirmé et un personnage parfaitement cosmopolite. Loewenthal, également russe, étudie à St-Pétersbourg, Kiev et Genève; spécialiste du système nerveux central et de l'appareil glandulaire, il est attiré à Lausanne sur la requête de Bugnion.

La Section propédeutique, embryon de Faculté, est d'abord logée dans la vieille caserne de la Cité, y accueillant modestement 13 premiers étudiants en 1881. Les premiers examens fédéraux de médecine lausannois ont lieu le 
15 octobre 1883 : les candidats, au nombre de 6, sont tous admis. En 1889/90, à la veille de la transformation, sur un total de 200 étudiants, la Faculté des sciences est la plus forte en nombre - 58, dont 34 en Section médicale -, devant le Droit. Dès octobre 1890, la nouvelle Faculté de médecine prend la tête, avec 63 étudiants sur 216 pour toute l'Académie. La médecine connaît alors une vogue extraordinaire, à rattacher évidemment aux grands succès de la bactériologie et, plus généralement, aux sensationnels aboutissements du siècle de la Science et du Progrès. La Section propédeutique est transférée en 1888 dans le bâtiment rénové de l'Ancienne douane de la Caroline, baptisé Ecole de médecine, en contre-bas du Champ-de-l'Air. Dès le début des cours à la Faculté, le 27 octobre 1890, l'anatomie pathologique dispose d'un institut de fortune au nord de l'hôpital; l'enseignement clinique est dispensé, outre à l'Hôpital cantonal, à l'Asile des aveugles de l'avenue de France, ainsi qu'à l'Asile d'aliénés de Cery. La première série d'examens professionnels complets a lieu en mars 1891. La même année sont délivrés deux diplômes de docteur en médecine, à des ressortissants des Grisons et du Piémont ${ }^{38}$.

Dans le contexte de l'hégémonie scientifique du Deuxième Reich (18711918), confortée par l'autorité du modèle universitaire humboldtien accordant la prééminence à l'expérimentation et à la recherche, appuyée par la puissance militaire et économique, la création de la Faculté de médecine de l'Université de Lausanne s'inspire naturellement des exemples d'outreRhin. La Commission Ruffy s'intéresse particulièrement à Innsbruck en Autriche, Erlangen et Würzburg en Bavière, Kiel et Rostock sur la Baltique $^{39}$. L'organisation germanique d'un corps enseignant composé de trois classes de maîtres est adoptée: professeurs ordinaires, extraordinaires et privat-docents. Système garantissant une constante émulation et combinant l'unicité de la médecine et de la chirurgie avec l'extension et la variété indispensables à l'essor de la science ${ }^{40}$. La loi fondatrice de 1890 confère à la Faculté 7 chaires de professeurs ordinaires, sur un total de 36 à l'Université. La voie normale de nomination est celle de l'appel par le Gouvernement, sur préavis de l'Université; le concours n'est envisagé qu'en deuxième ressort. Aussi Eugène Ruffy, entouré des conseils de sa Commission, complète-t-il l'enseignement propédeutique d'anatomie, histologie et physiologie en engageant, dans l'ordre, César Roux (1857-1934: chirurgie), Edouard de Cérenville (1843-1915: médecine), Marc Dufour (1843-1910: ophtalmologie) tous membres de la Commission préconsultative -, Siegfried Rabow (18481931: psychiatrie), Heinrich Stilling (1853-1911: anatomie pathologique), Louis Bourget (1856-1913: thérapeutique), Oscar Rapin (1847-1902 : obs- 


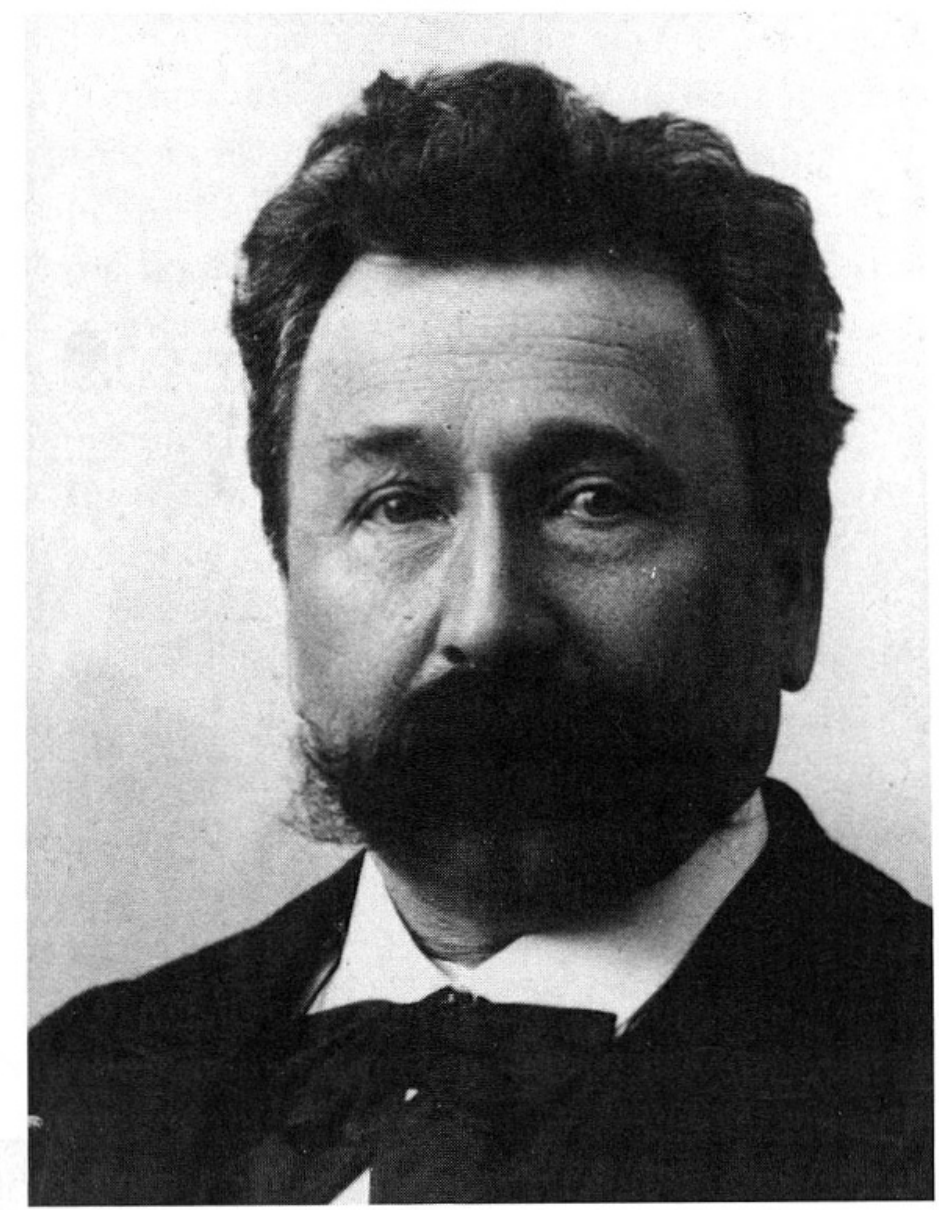

Le Dr Mare Dufour (1843-1910), ophtalmologue de renom international, premier doyen de la Faculté de médecine de Lausanne (Photographie Francis de Jongh, Musée historique de Lausanne).

tétrique); Jaques Larguier des Bancels (1844-1904: médecine légale) et Charles Nicolas (1846-1898: hygiène). Tous portent initialement le titre de professeur extraordinaire. A ce premier corps de douze enseignants sont bientôt adjoints les professeurs extraordinaires Emile Dind (1855-1932: dermatologie, 1891), également membre de la Commission Ruffy, et Paul Demiéville (1855-1947: policlinique, 1892). Les enseignements d'otologie et laryngologie, de pédiatrie et d'orthopédie sont donnés par les privat-docents Louis Secrétan (1891), Adolphe Combe et Louis Perret (1896) ${ }^{41}$.

L'ensemble des objets d'enseignement prévus par la Loi cantonale et le Règlement de Faculté sont ainsi pourvus, hormis l'histoire de la médecine, qu'Edouard de Cérenville, dans le cadre de la Commission Ruffy, avait fait inscrire dans le projet de loi le 14 avril 1890. Pressenti comme professeur 
d'histoire de la médecine par le chef du département, Henri-François Secrétan, médecin, fils du philosophe Charles, décline l'offre, malgré l'insistance de Roux et Stilling, «redoutant un travail complètement étranger à la nature pratique de [son] activité» ${ }^{42}$. Près de quarante ans s'écoulent ensuite, avant qu'André Guisan ne propose un cours libre; après quoi le pathologue de Francfort Edgar Goldschmid obtient charge de cours (1933).

Des douze premiers professeurs de 1890, huit sont vaudois (dont deux d'origine étrangère: Larguier et Roux), un fribourgeois d'origine russe (Herzen), un russe (Loewenthal) et deux prussiens (Rabow et Stilling). Mais tous, à l'exception de Larguier, sont issus de l'école allemande. Domination germanique incontestée et sans partage, gage de la féconde union du laboratoire et de la clinique.

La Faculté de médecine de l'Université de Lausanne, fruit du siècle d'or de la médecine et du fédéralisme vaudois, est contemporaine de la Faculté de Lyon (1877) ou de la très prestigieuse Johns Hopkins University School of Medicine de Baltimore (1893). Et la transformation de l'Académie s'inscrit bien dans le grand renouveau des Universités de la seconde partie du XIX

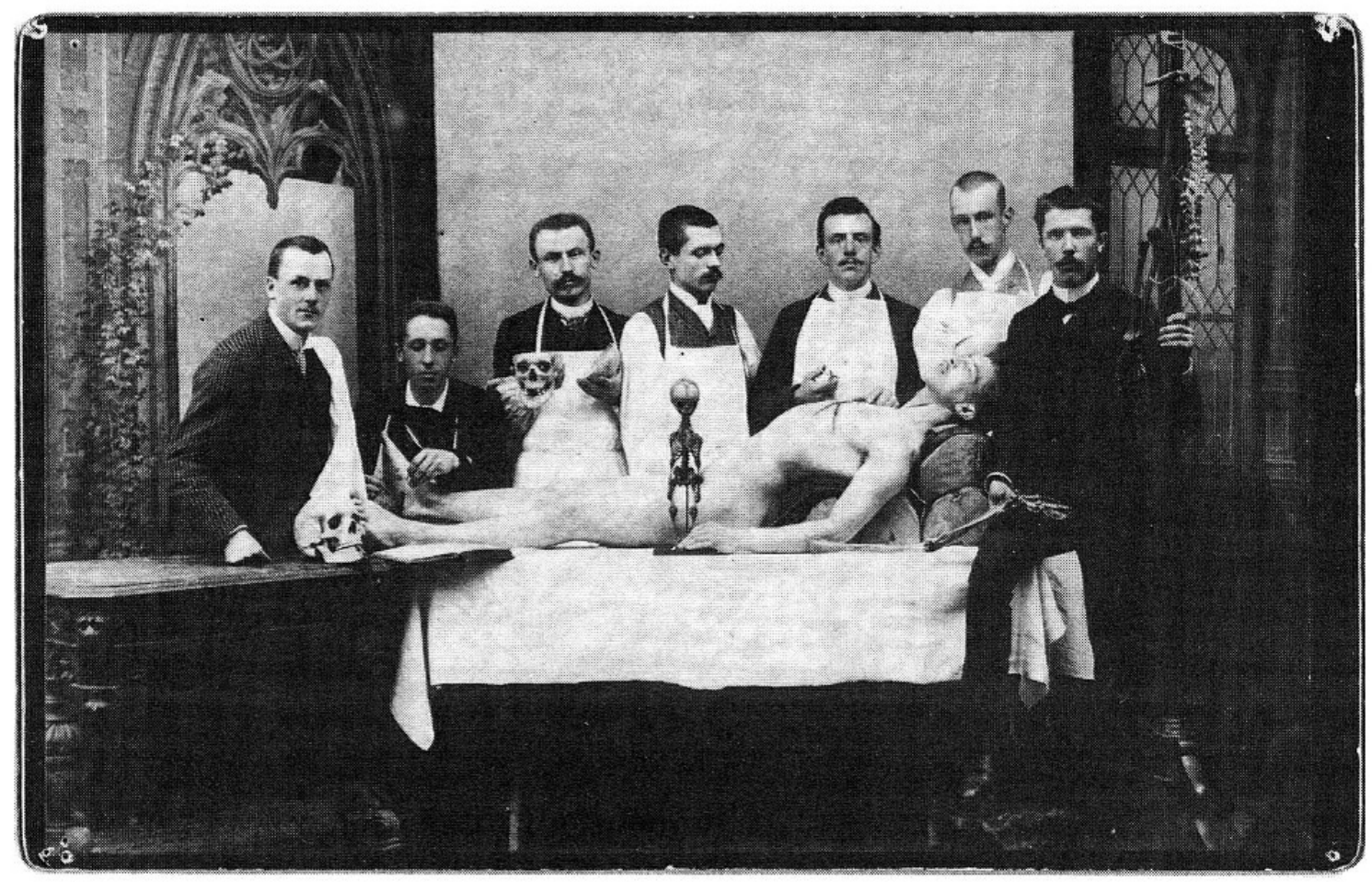

La leçon d'anatomie: canular estudiantin, Faculté de médecine de l'Université de Lausanne, vers 1890. De g. à dr. H. Vulliet, E. Olivier, Ch. Doukof, G. Rochaz, A. Ackermann, W. Luber, A. Chatelain; le «cadavre» est F. Paccaud (Musée de l'Elysée, Lausanne). 
siècle, sur le modèle allemand. Mais à Lausanne, 35000 habitants seulement en 1890, chef-lieu d'un vaste arrière-pays campagnard, le pari semble audacieux. Il intervient cependant à l'heure de la «Medicina triumphans» ${ }^{43}$ et au seuil de la Belle Epoque qui promeut Lausanne du rang de chef-lieu à celui de ville centre d'agglomération: doublement de la population résidente en deux décennies (1888-1910); doublement des actifs en une décennie, dans le bâtiment, le commerce, l'hôtellerie, les transports et communications (18901900); triplement de l'élément «étranger», non vaudois de souche, en un demi-siècle, dépassant $50 \%$ à la veille de la Grande Guerre. Age d'or du tourisme lémanique, chemin de fer Jura-Simplon, fièvre de la construction, plan d'extension, services industriels, tramways, socialisme, sans oublier l'Université, caractérisent l'extraordinaire densité des mutations au tournant du siècle ${ }^{44}$.

Les autorités sanitaires et le corps médical vaudois dans son ensemble, longtemps repliés sur eux-mêmes et farouchement opposés à toute intrusion fédérale dans le cadre professionnel, cèdent la barre aux autorités politiques et à une élite de médecins qui, dans le dernier tiers du $\mathrm{XIX}^{\mathrm{e}}$ siècle, face à l'intégration helvétique et à la concurrence genevoise, optent résolument pour une Faculté de médecine à essor rapide, soit cosmopolite, accueillant jusqu'à $84,4 \%$ d'étudiants étrangers en 1906/0745, démontrant à leur manière que l'attitude fédéraliste confine à l'ouverture... 
1 Olivier Eugène, Médecine et santé dans le Pays de Vaud au XVIIIe siècle: 1675-1798, Lausanne, 1939, p. $131 \mathrm{~s}$.

2 Ibid., p. 105 s.

3 Portmann Marie-Louise, Relations d'Auguste Tissot (1728-1797), médecin à Lausanne, avec le patriciat bernois, in Gesnerus 37, fasc. 1/2, 1980, p. 21-27.

4 Olivier Eugène, Jean-André Venel, d'Orbe (1740-1791). Maître accoucheur. Pionnier de l'orthopédie. Son caractère, son œuvre, sa famille, ses portraits, son sabot. Ses premiers successeurs vaudois, in L'éveil médical vaudois: Tissot, Venel, Mayor, études réunies et présentées par Guy Saudan, Lausanne, 1987, p. 51-103.

5 Lettre de François Verdeil au Petit Conseil, du 22 mars 1804: Archives cantonales vaudoises, Chaires de médecine, 1803-1836 (K XIII 24).

6 Olivier Eugène, Médecine et santé (op. cit.), p. $27 \mathrm{~s}$.

7 Ibid., p. 33 .

8 Lettre du 22 mars 1804 (op. cit.).

9 Olivier Eugène, Médecine et santé (op. cit.), p. $785 \mathrm{~s}$.

10 Müller Christian, Le Champ-de-l'Air, premier hôpital psychiatrique du Canton de Vaud, in Gesnerus, 37, 1980, p. 28-33.

11 Morax Jean-Mare, Statistique médicale du Canton de Vaud, Lausanne, 1899, p. $145 \mathrm{s.}$

12 Secrétan Claude, L'Hôpital de Cery: 1873-1973, Lausanne, 1973, 140 p.

13 Bolli Laurent, Chronique de l'Asile des aveugles de Lausanne: 1843-1943, Lausanne, 1944, 322 p. Van Muyden Berthold, Histoire de l'Asile des aveugles, 1844-1894, in Jubilé cinquantenaire de l'Asile des aveugles de Lausanne, Lausanne, 1894, p. 21-61.

14 Reynier Jean-Pierre de, L'Hôpital de l'enfance, de 1861 à 1979, in Revue médicale de la Suisse romande 100, 1980, p. 557-565.

15 Centième anniversaire de l'Hôpital orthopédique de la Suisse romande : 1876-1976, [par Jean Hugli et Louis Nicod], Lausanne, 1976, 136 p.

16 Saudan Guy, Dispensaire et Policlinique à Lausanne: 100 ans de médecine et société, in Revue médicale de la Suisse romande 109, 1989, p. 267-277.

17 Vincent Jules, Aux écoutes de la souffrance: histoire de l'Institution des diaconesses de Saint-Loup, 1842-1942, Lausanne, 1942, 302 p.

18 La Source: Ecole de gardes-malades, Lausanne, Suisse, 1859-1909, [préface de Charles Krafft], Lausanne, 1909, 84 p.

19 Biaudet Jean Charles, L'écharpe, le marteau et le tourniquet: Mathias Mayor, un chirurgien vaudois du début du XIXe siècle, in L'éveil médical vaudois (op. cit.), p. 105-174. Jean de La Harpe, docteur en médecine, à Lausanne [nécrologie], in Actes de la Société helvétique des sciences naturelles, Lausanne, 1878, p. 293-304. Guisan André, Les origines de la Société vaudoise de médecine, in Revue médicale de la Suisse romande 39, 1919, p.149-158 et 211-223.

20 Morax Jean-Marc (op. cit.), p. $179 \mathrm{~s}$.

21 Mollaret Henri H. et Brossollet Jacqueline, Alexandre Yersin, le vainqueur de la peste, Paris, $1985,320 \mathrm{p}$.

22 Meier Rolf, August Forel : 1848-1931, Arzt, Naturforscher, Sozialreformer, Zurich, 1986, $159 \mathrm{p}$. 
23 Del Regato Juan A., Gustave Roussy, in International Journal of Radiation: Oncology, Biology, Physics 15, 1988, p. 1223-1232.

24 Polla Louis, De saint Etienne au général Guisan: Louis Polla raconte la vie de cent personnages qui ont donné leur nom aux rues de Lausanne, Lausanne, 1981, p. 140-142.

25 Ganguillet Franz, Arztewesen in der Schweiz, Bâle, 1920, p.16s.

26 Delay Gustave, La Policlinique universitaire, in Recueil de travaux publiés à l'occasion du quatrième centenaire de l'Université de Lausanne: Faculté de médecine, Lausanne, 1937, p. 44.

27 Polla Louis (op. cit.), p. 138-140.

28 Exposé des motifs et projet de décret ratifiant la Convention conclue les 23 juin et 3 août 1888 entre l'Etat et la Commune de Lausanne pour l'affectation du legs de Rumine, Lausanne, 1888, p. 20.

29 Dufour Marc, Rapport présenté au Conseil communal de Lausanne au nom de la Commission du legs de Rumine, Lausanne, 1887, p. 31 s.

30 Bulletin des séances du Grand Conseil vaudois, débat sur la Convention de Rumine, 21 août 1888, p. 15.

31 Tissot Auguste, Essai sur les moyens de perfectionner les études de médecine, Lausanne, 1785, p. 15.

32 Bonjour Félix, Le percement du Simplon, Lausanne, 1906, 77 p. Lasserre André, La croissance et ses problèmes, in Histoire de Lausanne, publiée sous la direction de Biaudet Jean Charles, Lausanne, 1982, p. 306s.

33 Discours et leçons prononcés à l'ouverture des cours du Ier semestre de l'Université de Lausanne, Lausanne, 1891, p.4.

34 Tissot Laurent, De l'Académie à l'Université: 1869-1990, in De l'Académie à l'Université de Lausanne : 1537-1987, 450 ans d'histoire, Lausanne, 1987, p. 171.

35 Gazette de Lausanne, 12 mars 1890, et Revue, 13 mars 1890.

36 Taillens Jules, La fondation de la Faculté de médecine de Lausanne, in Cinquantenaire de la Faculté de médecine de Lausanne: 1890-1940, Lausanne, 1940, p. 48.

37 Olivier Eugène, 1868-1900: quelques dates et notes [manuscrit], p.6-7; Bibliothèque cantonale et universitaire, Lausanne, Département des manuscrits (IS 1905/134).

38 Compte rendu du Conseil d'Etat, Etat de Vaud, Lausanne.

39 Exposé des motifs (op. cit.), p. 22.

40 Jaccoud Sigismond, De l'organisation des Facultés de médecine en Allemagne: rapport présenté à Son Excellence le Ministre de l'instruction publique, le 6 octobre 1863, Paris, 1864, 174 p.

41 Bonnard Jean, Université de Lausanne: 1890-1901, in Programme des cours de l'Université de Lausanne, Lausanne, Hiver 1901/02.

42 Archives cantonales vaudoises (K XIII 252 C et 278/1).

43 Lichtenthaeler Charles, Geschichte der Medizin, Cologne, 1975, vol. 2, p. 561-566.

44 Lasserre André (op. cit.).

45 Catalogue des étudiants de l'Université de Lausanne, Lausanne. 


\title{
Summary
}

\section{The Faculty of Medicine of Lausanne University (1890) : a political challenge}

The Lausanne Faculty of Medicine was originally founded for political rather than medical reasons: Vaud's accession to cantonal sovereignty made possible the call, in 1804, for a medical school, and the canton's federalist convictions brought it into being in 1890 . - In the last quarter of the nineteenth century, Lausanne had to assert its own position between the patrician power of Bern, meanwhile elevated to federal capital, and industrious Geneva. Discussions centred on the siting of new railway lines and of facilities for higher education. It was thereupon decided that Vaud's cantonal capital should become a major intersection for rail traffic and a university city. Then, a medical faculty was created in Geneva (1876) and federal medical examination was instituted (1877). These two developments had a catalytic effect: after introductory courses had begun in 1881, a complete medical faculty was established in Lausanne in 1890, enabling the former Academy inherited from the days of Bernese administration to be transformed into a fullscale university (1890).

\section{Zusammenfassung}

\section{Die Medizinische Fakultät der Universität Lausanne (1890) : ein politischer Erfolg.}

Eher politische als medizinische Ansprüche haben zur Gründung der medizinischen Fakultät in Lausanne beigetragen : die Kantonssouveränität erlaubt 1804 das Projekt einer Hohen Schule; der waadtländische Föderalismus trägt 1890 zu dessen Verwirklichung bei. - Im letzten Viertel des 19. Jahrhunderts muss sich Lausanne zwischen der alten Patrizierherrschaft und neuen Bundeshauptstadt Bern einerseits, dem geschäftstüchtigen Genf andererseits, einen Platz erkämpfen. Strategische Punkte der Verhandlungen sind Eisenbahnlinien und Standort der Hochschule: die waadtländische Hauptstadt wird zugleich Bahnknotenpunkt und Universitätsstadt. Die Liberalen führen die von Bern geprägte Akademie in weltliche Hände über, die Radikalen bringen die Wissenschaften zum Aufblühen. Die Gründung einer medizinischen Fakultät in Genf (1876) und die Einführung der eidgenössischen Medizinalprüfungen (1877) wirken als Katalysator: nach mehrjährigem Bestehen eines propädeutischen Unterrichts (seit 1881) entsteht 1890 in Lausanne eine vollständige medizinische Fakultät und ermöglicht den Übergang von der ehemaligen Akademie zur Universität.

\author{
Guy Saudan \\ Institut universitaire d'histoire de la médecine et de la santé publique \\ C.P 139 \\ CH-1000 Lausanne 4
}

